\title{
EFEITOS DA LUZ E TEMPERATURA NA GERMINAÇÃO DE SEMENTES DE QUATRO ESPÉCIES DE PLANTAS DANINHAS DO GÊNERO Digitaria ${ }^{1}$
}

\author{
VITOR HENRIQUE VAZ MONDO2; SAUL JORGE PINTO DE CARVALHO; \\ ANA CAROLINA RIBEIRO DIAS²; JÚLIO MARCOS FILHO ${ }^{3}$
}

\begin{abstract}
RESUMO - O Brasil é o país das Américas com maior número de espécies nativas do gênero Digitaria, conhecidas como plantas daninhas, constituindo problemas em várias culturas de expressão econômica e encontradas em várias regiões do território brasileiro, sob ampla variação de ambientes. Este trabalho foi desenvolvido com o objetivo de avaliar o efeito da temperatura e presença de luz na germinação de sementes de quatro espécies de plantas daninhas do gênero Digitaria. O experimento foi conduzido em câmaras de germinação, em esquema fatorial $4 \times 2$, incluindo quatro temperaturas $\left(25^{\circ} \mathrm{C}\right.$ constantes e alternâncias de $20-30^{\circ} \mathrm{C}, 20-35^{\circ} \mathrm{C}$ e $15-35^{\circ} \mathrm{C}$ ) em fotoperíodo de $8 \mathrm{~h}$ e na ausência de luz. Foram estudadas sementes das espécies Digitaria bicornis (Lam.) Roem. \& Schult., Digitaria ciliaris (Retz.) Koel. e Digitaria horizontalis Willd de nome comum capim-colchão e, Digitaria insularis (L.) Fedde de nome comum capim-amargoso. Foram avaliadas a porcentagem e a velocidade de germinação até os 21 dias após o início do teste de germinação. Constatou-se que as exigências de temperatura e luz variam de acordo com a espécie, sendo que $D$. bicornis e $D$. horizontalis necessitam de luz para a germinação, o que não se verifica para $D$. ciliaris e a $D$. insularis. As temperaturas mais adequadas para a germinação das sementes são $20-35^{\circ} \mathrm{C}$, para $D$. bicornis e $D$. ciliaris e $20-35^{\circ} \mathrm{C}$ ou $15-35^{\circ} \mathrm{C}$, para $D$. horizontalis e $D$. insularis.
\end{abstract}

Termos para indexação: plantas daninhas, capim-colchão, capim-amargoso, análise de sementes.

\section{LIGHT AND TEMPERATURE EFFECTS ON THE SEED GERMINATION OF FOUR Digitaria WEED SPECIES}

\begin{abstract}
Brazil has the largest number of native species of Digitaria in the Americas. This genus is considered as a weed causing problems in various economic crops and can be found throughout Brazil in many different ecological situations. The object of this research was to evaluate the effects of light and temperature on the seed germination of four Digitaria weed species. The experiment was conducted in germination chambers using a factorial $4 \times 2$ scheme of treatments under four temperatures $\left(25^{\circ} \mathrm{C}\right.$ constant, alternating $20-30^{\circ} \mathrm{C}, 20-35^{\circ} \mathrm{C}$ and $\left.15-35^{\circ} \mathrm{C}\right)$ with alternate light cycles (8h light/16h darkness) or absolute darkness. The weed species studied were Digitaria horizontalis Willd., D. bicornis (Lam.) Roem. \& Schult., D.ciliaris (Retz.) Koel. and D. insularis (L.) Fedde. The percentage and speed of germination were evaluated up to 21 days after the start of the germination test. The four species showed differing seed physiological responses. $D$. bicornis and $D$. horizontalis required light for germination while $D$. ciliaris and $D$. insularis did not. The best temperatures for germination are $20-35^{\circ} \mathrm{C}$ for $D$. bicornis and D. ciliaris, and $20-35^{\circ} \mathrm{C}$ or $15-35^{\circ} \mathrm{C}$ for $D$. horizontalis and $D$. insularis.
\end{abstract}

Index terms: weed seeds, crabgrass, seed analysis.

${ }^{1}$ Submetido em 17/11/2008. Aceito para publicação em 26/11/2009.

${ }^{2}$ Eng. Agr., pós-graduando em Fitotecnia, USP/ESALQ, e-mail: vhvmondo@ yahoo.com.br; sjpcarvalho@yahoo.com.br; acrdias@esalq.usp.br
${ }^{3}$ Eng. Agr., Dr., Professor Titular, Departamento de Produção Vegetal, USP/ ESALQ; bolsista do CNPq; Caixa Postal 09; CEP 13418-900; Piracicaba, SP; jmarcos@esalq.usp.br 


\section{INTRODUÇÃO}

O gênero Digitaria compreende cerca de 300 espécies de plantas distribuídas em diferentes regiões do mundo, tanto tropicais quanto subtropicais (Canto-Dorow, 2001). O Brasil é o país das Américas com maior diversidade de espécies desse gênero, tendo sido constatada a presença de 26 espécies nativas e de 12 exóticas. Destas, 13 foram identificadas somente no Estado de São Paulo. Conhecidas popularmente como capim-colchão e capim-amargoso, são plantas de desenvolvimento rápido e agressivo em áreas cultivadas e relatadas como problema em mais de 60 países, infestando mais de 30 culturas de importância econômica; no Brasil, podem ocorrer regularmente em várias culturas "de primavera" ou "de verão" (Kissmann, 1997). Atualmente, têm sido consideradas como uma das principais espécies de plantas daninhas que infestam os cultivos de cana-de-açúcar (Dias et al., 2007).

Os programas de manejo integrado de plantas daninhas têm como uma das maiores limitações a carência de conhecimentos sobre biologia e ecologia (Fernandez, 1982), considerados essenciais para o desenvolvimento de sistemas de manejo viáveis sob os pontos de vista econômicos e ambientais (Bhowmik, 1997). O conhecimento de aspectos relacionados à germinação, como temperatura, causas da dormência e a profundidade máxima que possibilita a germinação das plantas daninhas, associadas à adoção de práticas adequadas de manejo como, por exemplo, a determinação do momento ótimo para a aplicação de herbicidas em pós-emergência (Guo e Al-Khatib, 2003) são informações de grande importância para a aplicação de sistemas viáveis de manejo integrado.

A germinação das sementes é regulada pela interação de seu estado fisiológico e das condições de ambiente, sendo que cada espécie vegetal exige um conjunto de requisitos específicos quanto à disponibilidade de água, temperatura, luz e profundidade de semeadura, para a ocorrência do processo de germinação. A temperatura é considerada ótima para a germinação das sementes quando permite a expressão do potencial máximo de germinação em menor período de tempo (Popinigis, 1985; Mayer e Poljakoff Mayber, 1989). Normalmente, essa temperatura está relacionada à temperatura da região de origem geográfica da espécie, considerando a época favorável para a germinação (Andrade et al., 2000). Dessa forma, existem espécies cujo processo germinativo é favorecido por temperatura constante (Varela et al., 1999; Sousa et al., 2000; Silva, 2001), por alternância de temperatura (Santos e Aguiar, 2000; Lopes e Soares, 2003) ou por um intervalo amplo de temperatura (Nassif e Perez, 2000; Silva et al., 2002).

A luz é outro fator importante para a germinação das sementes. Tanto a intensidade, o comprimento de onda quanto o fotoperíodo são variáveis conhecidas por exercerem efeito sobre a germinação de sementes que possuem dormência (Copeland e McDonald, 2001). Quando as sementes necessitam da presença de luz para germinar, elas são denominadas fotoblásticas positivas; quando necessitam da ausência de luz, fotoblásticas negativas; e quando a luz não interfere no processo germinativo, fotoblásticas neutras ou não fotoblásticas (Mayer e Poljakoff Mayber, 1989; Vázquez-Yanes e Orozco-Segovia, 1993).

$\mathrm{Na}$ realidade a necessidade de temperaturas alternadas e de luz para a germinação de sementes são exigências para a superação da dormência das sementes. Assim, caso as condições não sejam as ideais para a germinação, as sementes podem permanecer vivas nos solos por longos períodos (Steckel et al., 2004).

O conhecimento das exigências ambientais para germinação das sementes de espécies de plantas daninhas, ainda não disponível para várias espécies, é fundamental para a interpretação do seu comportamento ecológico no campo, além de possibilitar o desenvolvimento de estratégias de redução do banco de sementes nas áreas cultivadas (Souza Filho, 2006).

Neste contexto, o presente trabalho teve o objetivo de avaliar o efeito de diferentes temperaturas e de luz na germinação de sementes de quatro espécies de plantas daninhas do gênero Digitaria.

\section{MATERIAL E MÉTODOS}

A pesquisa foi realizada no Laboratório de Análise de Sementes, do Departamento de Produção Vegetal da Escola Superior de Agricultura "Luiz de Queiroz", Universidade de São Paulo, em Piracicaba - SP, entre março e junho de 2008.

Foram estudadas quatro espécies do gênero Digitaria: Digitaria horizontalis Willd., Digitaria bicornis (Lam.) Roem. \& Schult. e Digitaria ciliaris (Retz.) Koel., de nome comum capim-colchão e, Digitaria insularis (L.) Fedde, de nome comum capim-amargoso. As sementes foram coletadas de 20 plantas em infestações naturais localizadas em áreas agrícolas do Departamento de Produção Vegetal (USP/ESALQ), no mês de março de 2008, constituindo lotes entre cinco e dez gramas de sementes de cada espécie. As panículas foram identificadas segundo chave dicotômica (Dias et al., 2007) e armazenadas em câmara a $20^{\circ} \mathrm{C}$ e 50 $60 \%$ de umidade relativa do ar até o momento da instalação 
do experimento.

Realizou-se o teste de germinação para cada uma das quatro espécies coletadas, em delineamento experimental inteiramente casualizado com quatro repetições, segundo esquema fatorial $4 \times 2$, ou seja, quatro temperaturas: $25^{\circ} \mathrm{C}$ (constante) e alternâncias $20-30{ }^{\circ} \mathrm{C}, 20-35^{\circ} \mathrm{C}$ e $15-35^{\circ} \mathrm{C}$; e duas condições de luz: ausência de luz ou fotoperíodo diário de oito horas, coincidindo com a temperatura mais alta da alternância.

As temperaturas propostas para germinação das sementes foram baseadas nas Regras para Análise de Sementes (BRASIL, 1992), onde estão indicadas as temperaturas de 20$35^{\circ} \mathrm{C}$ e $15-35^{\circ} \mathrm{C}, 15^{\circ} \mathrm{C}$ e $20^{\circ} \mathrm{C}$ de amplitude de temperatura, para as espécies de Digitaria spp.; foi, também, incluída uma condição intermediária de amplitude de temperatura $\left(20-30{ }^{\circ} \mathrm{C}\right)$ e outra temperatura constante $\left(25^{\circ} \mathrm{C}\right)$, comumente utilizadas em germinadores de laboratórios de análise de sementes.

A germinação foi conduzida em caixas de plástico (11,0 x $11,0 \times 3,0 \mathrm{~cm})$ transparentes para os testes com presença de luz ou pretas para os testes em ausência de luz. Para cada repetição do teste, foram dispostas 50 sementes sobre duas folhas de papel mata-borrão $(10,5 \times 10,5 \mathrm{~cm})$ previamente umedecidas com quantidade de água correspondente a 2,5 vezes a massa do papel seco. Para os testes com ausência de luz, as parcelas foram semeadas apenas na presença de luz verde, para evitar interferência da luz no processo de germinação (Noronha et al., 1978). Em seguida, as caixas foram acondicionadas no interior de sacos plásticos transparentes, a fim de evitar a perda de água, e colocadas em câmaras B.O.D.s com controle de temperatura e de disponibilidade de luz, de acordo com cada tratamento. Tais câmaras eram equipadas com fonte de luz frontal composta por quatro lâmpadas fluorescentes brancas de $15 \mathrm{w}$ cada (GE, “daylight”) e, realizou-se o rodízio das caixas plásticas diariamente dentro de cada câmara, afim de oferecer de forma homogênea intensidade luminosa para todas as repetições.

A interpretação dos testes de germinação foi realizada diariamente até os 21 dias após a semeadura, computandose como plântulas normais as que possuíam as partes do embrião convenientemente desenvolvidas. Para as parcelas conduzidas em ausência de luz, as avaliações foram realizadas apenas na presença de luz verde, pela razão já exposta. Foi calculado o índice de velocidade de germinação (IVG), segundo fórmula descrita por Maguire (1962) e a porcentagem total de germinação para cada tratamento.

Para a análise dos dados aplicou-se teste F. Na ocorrência de efeitos significativos, as médias foram comparadas pelo teste de Tukey, com 5\% de significância. Os dados percentuais foram previamente transformados por

$$
\text { arcen } \sqrt{\frac{x}{100}}
$$

\section{RESULTADOS E DISCUSSÃO}

A aplicação do teste $\mathrm{F}$ na análise da variância indicou a interação fatorial das diferentes condições de luz e temperatura para as quatro espécies de plantas daninhas, consideradas como quatro experimentos independentes (Tabelas 1 a 4). Em um exame conjunto das Tabelas 1 a 4 observa-se que, para todas as espécies avaliadas, sob temperatura de $25{ }^{\circ} \mathrm{C}$ constante a porcentagem e a velocidade de germinação foram inferiores às verificadas sob as temperaturas alternadas de 20-35 ${ }^{\circ} \mathrm{C}$ e $15-35^{\circ} \mathrm{C}$; estas, de um modo geral, foram as mais adequadas para a germinação das sementes.

TABELA 1. Porcentagem e índice de velocidade de germinação (IVG) de sementes de Digitaria bicornis expostas a diferentes condições de temperatura e luz, aos 21 dias após instalação.

\begin{tabular}{|c|c|c|c|c|}
\hline \multirow{2}{*}{$\begin{array}{c}\text { Temperaturas } \\
\left({ }^{\circ} \mathrm{C}\right)\end{array}$} & \multicolumn{2}{|c|}{ Germinação $(\%)^{1}$} & \multicolumn{2}{|c|}{$\mathrm{IVG}^{1}$} \\
\hline & Luz & Escuro & Luz & Escuro \\
\hline 25 & $0 \mathrm{Ca}$ & $0 \mathrm{Aa}$ & $0,00 \mathrm{Ca}$ & $0 \mathrm{Aa}$ \\
\hline $20-30$ & $50 \mathrm{Ba}$ & $0 \mathrm{Ab}$ & $3,19 \mathrm{Ba}$ & $0 \mathrm{Ab}$ \\
\hline $20-35$ & $78 \mathrm{Aa}$ & $1 \mathrm{Ab}$ & $6,52 \mathrm{Aa}$ & $0 \mathrm{Ab}$ \\
\hline \multirow[t]{3}{*}{$15-35$} & $44 \mathrm{Ba}$ & $0 \mathrm{Ab}$ & $3,30 \mathrm{Ba}$ & $0 \mathrm{Ab}$ \\
\hline & C.V. $(\%)=21,82$ & $\mathrm{~F}_{(\text {Temp x Luz) }}=67,45^{*}$ & C.V. $(\%)=26,91$ & $\mathrm{~F}_{(\text {Temp x Luz) }}=69,75^{*}$ \\
\hline & $\operatorname{DMS}_{(\text {linha) }}=6,19$ & $\mathrm{DMS}_{\text {(coluna) }}=8,27$ & $\mathrm{DMS}_{\text {(linha) }}=0,65$ & $\mathrm{DMS}_{\text {(coluna) }}=0,86$ \\
\hline
\end{tabular}

*Valor de F significativo no nível de 5\% de probabilidade; ${ }^{1}$ Médias seguidas por letras iguais, maiúsculas na coluna ou minúsculas na linha, não diferem entre si segundo teste de Tukey, com 5\% de significância. 
TABELA 2. Porcentagem e índice de velocidade de germinação (IVG) de sementes de Digitaria ciliaris expostas a diferentes condições de temperatura e luz, aos 21 dias após instalação.

\begin{tabular}{|c|c|c|c|c|}
\hline \multirow{2}{*}{$\begin{array}{c}\text { Temperaturas } \\
\qquad\left({ }^{\circ} \mathrm{C}\right)\end{array}$} & \multicolumn{2}{|c|}{ Germinação $(\%)^{1}$} & \multicolumn{2}{|c|}{$\mathrm{IVG}^{1}$} \\
\hline & Luz & Escuro & Luz & Escuro \\
\hline 25 & $36 \mathrm{Ba}$ & $0 \mathrm{Cb}$ & $2,54 \mathrm{Ba}$ & $0,06 \mathrm{Cb}$ \\
\hline $20-30$ & $48 \mathrm{Ba}$ & $4 \mathrm{Cb}$ & $3,90 \mathrm{Bb}$ & $0,35 \mathrm{Cb}$ \\
\hline $20-35$ & $80 \mathrm{Aa}$ & $78 \mathrm{Aa}$ & $13,50 \mathrm{Aa}$ & $9,03 \mathrm{Ab}$ \\
\hline \multirow[t]{3}{*}{$15-35$} & $92 \mathrm{Aa}$ & $48 \mathrm{Bb}$ & $11,98 \mathrm{Aa}$ & $3,28 \mathrm{Bb}$ \\
\hline & C.V. $(\%)=19,92$ & $\mathrm{~F}_{(\text {Temp x Luz) }}=6,85^{*}$ & C.V. $(\%)=5,58$ & $\mathrm{~F}_{(\text {Temp x Luz) }}=12,04 *$ \\
\hline & $\operatorname{DMS}_{(\text {linha) }}=12,26$ & $\mathrm{DMS}_{(\text {coluna) }}=16,38$ & $\operatorname{DMS}_{\text {(linha) }}=1,62$ & $\operatorname{DMS}_{\text {(coluna) }}=2,17$ \\
\hline
\end{tabular}

*Valor de F significativo no nível de 5\% de probabilidade; ${ }^{1}$ Médias seguidas por letras iguais, maiúsculas na coluna ou minúsculas na linha, não diferem entre si segundo teste de Tukey, com 5\% de significância.

TABELA 3. Porcentagem e índice de velocidade de germinação (IVG) de sementes de Digitaria horizontalis expostas a diferentes condições de temperatura e luz, aos 21 dias após instalação.

\begin{tabular}{|c|c|c|c|c|}
\hline \multirow{2}{*}{$\begin{array}{l}\text { Temperaturas } \\
\left({ }^{\circ} \mathrm{C}\right)\end{array}$} & \multicolumn{2}{|c|}{ Germinação $(\%)^{1}$} & \multicolumn{2}{|c|}{$\mathrm{IVG}^{1}$} \\
\hline & Luz & Escuro & Luz & Escuro \\
\hline 25 & $4 \mathrm{Ca}$ & $1 \mathrm{Ba}$ & $0,77 \mathrm{Ba}$ & $0,20 \mathrm{Aa}$ \\
\hline $20-30$ & $24 \mathrm{Ba}$ & $1 \mathrm{Bb}$ & $2,41 \mathrm{Ba}$ & $0,14 \mathrm{Aa}$ \\
\hline $20-35$ & $45 \mathrm{Aa}$ & $6 \mathrm{ABb}$ & $6,67 \mathrm{Aa}$ & $0,69 \mathrm{Ab}$ \\
\hline \multirow[t]{3}{*}{$15-35$} & $35 \mathrm{ABa}$ & $9 \mathrm{Ab}$ & $5,35 \mathrm{Aa}$ & $0,84 \mathrm{Ab}$ \\
\hline & C.V. $(\%)=28,65$ & $\mathrm{~F}_{(\text {Temp x Luz) }}=5,27 *$ & C.V. $(\%)=58,04$ & $\mathrm{~F}_{(\text {Temp x Luz) }}=7,48^{*}$ \\
\hline & $\mathrm{DMS}_{(\text {linha) }}=8,28$ & $\mathrm{DMS}_{\text {(coluna) }}=11,06$ & $\mathrm{DMS}_{(\text {linha) }}=1,81$ & $\mathrm{DMS}_{\text {(coluna) }}=2,41$ \\
\hline
\end{tabular}

*Valor de F significativo no nível de $5 \%$ de probabilidade; ${ }^{1}$ Médias seguidas por letras iguais, maiúsculas na coluna ou minúsculas na linha, não diferem entre si segundo teste de Tukey, com 5\% de significância.

TABELA 4. Porcentagem e índice de velocidade de germinação (IVG) de sementes de Digitaria insularis expostas a diferentes condições de temperatura e luz, aos 21 dias após instalação.

\begin{tabular}{|c|c|c|c|c|}
\hline \multirow{2}{*}{$\begin{array}{l}\text { Temperaturas } \\
\left({ }^{\circ} \mathrm{C}\right)\end{array}$} & \multicolumn{2}{|c|}{ Germinação $(\%)^{1}$} & \multicolumn{2}{|c|}{$\mathrm{IVG}^{1}$} \\
\hline & Luz & Escuro & Luz & Escuro \\
\hline 25 & $67 \mathrm{Ba}$ & $33 \mathrm{Cb}$ & $13,18 \mathrm{Ba}$ & $8,01 \mathrm{Cb}$ \\
\hline $20-30$ & $96 \mathrm{Aa}$ & $64 \mathrm{Bb}$ & $23,60 \mathrm{Aa}$ & $13,78 \mathrm{Bb}$ \\
\hline $20-35$ & $97 \mathrm{Aa}$ & 97 Aa & $23,45 \mathrm{Aa}$ & $23,53 \mathrm{Aa}$ \\
\hline \multirow[t]{3}{*}{$15-35$} & $94 \mathrm{Aa}$ & $95 \mathrm{Aa}$ & $21,25 \mathrm{Aa}$ & $22,98 \mathrm{Aa}$ \\
\hline & C.V. $(\%)=9,06$ & $\mathrm{~F}_{(\text {Temp x Luz) }}=9,89 *$ & C.V. $(\%)=7,16$ & $\mathrm{~F}_{(\text {Temp x Luz) }}=30,67^{*}$ \\
\hline & $\mathrm{DMS}_{\text {(linha) }}=8,82$ & $\operatorname{DMS}_{\text {(coluna) }}=11,78$ & $\operatorname{DMS}_{(\text {linha) }}=1,96$ & $\operatorname{DMS}_{\text {(coluna) }}=2,61$ \\
\hline
\end{tabular}

\footnotetext{
*Valor de F significativo no nível de $5 \%$ de probabilidade; ${ }^{1}$ Médias seguidas por letras iguais, maiúsculas na coluna ou minúsculas na linha, não
} diferem entre si segundo teste de Tukey, com 5\% de significância. 
Sementes de várias espécies requerem variação diária da temperatura para a obtenção da germinação ótima. Tal periodicidade é comum e parece ter influência mais pronunciada em espécies que não passaram por trabalho intenso de domesticação, como várias espécies florestais e gramíneas nativas, que apresentam germinação mais elevada quando expostas a temperaturas alternadas. A necessidade dessa variação de temperatura durante a germinação está associada com a dormência de sementes; no entanto, também, existem relatos em que podem acelerar o processo de germinação de sementes não-dormentes (Copeland e McDonald, 2001).

Realmente, para algumas espécies tropicais, é conhecido que a alternância das temperaturas é considerada a melhor condição para a germinação das sementes, como constataram Gomes e Bruno (1992) para Bixa orellana (L.), Castellani e Aguiar (1998) para Trema micrantha (L.) e Carvalho e Christoffoleti (2007) para espécies do gênero Amaranthus.

Para as sementes de $D$. bicornis, os resultados mais favoráveis, para porcentagem e velocidade de germinação, foram obtidos no regime de temperaturas alternadas 20-35 ${ }^{\circ} \mathrm{C}$ (Tabela 1). Nessa tabela, ao se compararem resultados obtidos sob iluminação com os obtidos na ausência de luz, constatou-se efeito positivo da presença de luz na germinação das sementes, ou seja, há ocorrência de maior porcentagem e velocidade de germinação das sementes dessa espécie.

Na Tabela 2 são apresentados os resultados referentes à $D$. ciliaris. Neste caso, a porcentagem e a velocidade de germinação mais elevadas foram obtidas nos regimes de temperaturas alternadas $20-35{ }^{\circ} \mathrm{C}$ e $15-35^{\circ} \mathrm{C}$, em presença de luz. Aparentemente o aumento da amplitude térmica diária possibilitou melhores condições para a germinação; entretanto, observou-se que na ausência de luz apenas o intervalo de temperatura $20-35{ }^{\circ} \mathrm{C}$ manteve os mesmos resultados, enquanto para a temperatura $15-35{ }^{\circ} \mathrm{C}$ houve decréscimo acentuado para ambos os parâmetros. Para as espécies que necessitam de temperaturas alternadas para a germinação das sementes, a amplitude de variação da temperatura aparentemente é mais importante do que os valores absolutos das temperaturas (Murdoch et al., 1989). McDonald et al. (1994) reportaram que essa diferença foi de $10{ }^{\circ} \mathrm{C}$ para a germinação da maioria das gramíneas de estações frias, no entanto, a amplitude térmica para a espécie D. ciliaris foi de $15^{\circ} \mathrm{C}$. Com base nesses resultados, identificou-se que a temperatura foi mais importante que a luminosidade sobre a germinação dessas sementes.

Examinando-se a Tabela 3, para D. horizontalis, verifica- se que, em presença de luz, a porcentagem e velocidade de germinação foram mais elevadas sob temperaturas alternadas de $20-35{ }^{\circ} \mathrm{C}$ e $15-35{ }^{\circ} \mathrm{C}$, não diferentes entre si. Observouse, também, que a ausência de luz afetou negativamente a germinação das sementes dessa espécie, demonstrando a importância da presença de luz para a germinação dessas sementes, indicando característica fotoblástica positiva.

$\mathrm{Na}$ Tabela 4 são observados os resultados obtidos para sementes de $D$. insularis. As temperaturas $20-30{ }^{\circ} \mathrm{C}, 20-35^{\circ} \mathrm{C}$ e $15-35^{\circ} \mathrm{C}$ foram as que resultaram em maiores porcentagens e velocidades de germinação, sempre associadas à presença de luz. Ao mesmo tempo, para temperaturas $20-35^{\circ} \mathrm{C}$ e 15 $35^{\circ} \mathrm{C}$, a ausência de luz não interferiu na germinação das sementes.

Casos de interação das condições de luz e temperatura foram relatados por Taylorson e Hendricks (1972) e Takaki et al. (1985), segundo os quais a temperatura pode causar alterações da sensibilidade da semente a baixos níveis de Fve pré-existentes, favorecendo a germinação no escuro, fato que pode explicar as elevadas porcentagens de germinação das espécies $D$. insularis e $D$. ciliaris no escuro, sob temperaturas mais elevadas. De forma semelhante, Carvalho et al. (2005) estudaram aspectos relacionados à germinação do capim-branco (Chloris polydactyla), identificando maiores percentuais de germinação em presença de luz, interagindo com a alternância de temperatura.

As Regras para Análise de Sementes (Brasil, 1992) indicam intervalos de $15-35^{\circ} \mathrm{C}$ ou $20-35^{\circ} \mathrm{C}$ para a germinação de sementes de Digitaria spp. Realmente, ao comparar os resultados para as quatro espécies analisadas no presente trabalho, os regimes mais adequados de temperatura foram próximos dessa recomendação. Porém, para cada espécie, existem condições mais adequadas para a germinação das sementes, sendo as características fisiológicas dessas espécies realmente diferentes, principalmente quanto ao efeito presença ou ausência de luz sobre a germinação.

\section{CONCLUSÕES}

As espécies D. bicornis, D. ciliaris, D. horizontalis e $D$. insularis apresentam exigências diferentes quanto à temperatura e luz para a germinação das sementes. As sementes de $D$. bicornis e $D$. horizontalis necessitam de luz para a germinação, o que não se verifica para $D$. ciliaris e $D$. insularis.

As condições de temperatura mais adequadas para a germinação são $20-35^{\circ} \mathrm{C}$ para $D$. bicornis e $D$. ciliaris e 20$35^{\circ} \mathrm{C}$ ou $15-35^{\circ} \mathrm{C}$ para $D$. horizontalis e D. insularis. 


\section{REFERÊNCIAS}

ANDRADE, A.C.S.; SOUZA, A.F.; RAMOS, F.N.; PEREIRA, T.S.; CRUZ, A.P.M. Germinação de sementes de jenipapo: temperatura, substrato e morfologia do desenvolvimento pós-seminal. Pesquisa Agropecuária Brasileira, v.35, n.3, p.609-615, 2000.

BHOWMIK, P.C. Weed biology: importance to weed management. Weed Science, v.45, n.33, p.349-356, 1997.

BRASIL, Ministério da Agricultura e Reforma Agrária. Secretaria Nacional de Defesa Agropecuária. Departamento Nacional de Produção Vegetal. Coordenação de Laboratório Vegetal. Regras para análise de sementes. Brasília, DF, 1992. 365p.

CANTO-DOROW, T.S. Digitaria Heister ex Haller. In: WANDERLEY, M.G.L.; SHEPHERD, G.J.; GIULIETTI, A.M. (Ed.) Flora fanerogâmica do Estado de São Paulo. São Paulo: HUCITEC, 2001. p.143-150.

CARVALHO, S.J.P.; NICOLAI, M.; LÓPEZ-OVEJERO, R.F.; CHRISTOFFOLETI, P.J. Influência da luz, temperatura e profundidade da semente no solo sobre a germinação e emergência do capim-branco (Chloris polydactyla). Boletim Informativo - SBCPD, v.12, n.2, p.11-15, 2005.

CARVALHO, S.J.P.; CHRISTOFFOLETI, P.J. Influência da luz e da temperatura na germinação de cinco espécies de plantas daninhas do gênero Amaranthus. Bragantia, v.66, n.4, p.527533, 2007.

CASTELLANI, E.D.; AGUIAR, I.B. Condições preliminares para a germinação de sementes de candiúba (Trema micrantha (L.) Blume). Revista Brasileira de Engenharia Agrícola e Ambiental, v.2, n.1, p.80-83, 1998.

COPELAND, L.O.; McDONALD, M.B. Principles of seed science and technology. 4.ed. Boston, MA: Kluwer Academic Publishers, 2001. 467p.

DIAS, A.C.R.; CARVALHO, S.J.P.; NICOLAI, M.; CHRISTOFFOLETI, P.J. Problemática da ocorrência de diferentes espécies de capim-colchão (Digitaria spp.) na cultura da cana-de-açúcar. Planta Daninha. v.25, n.3, p. 489-499, 2007.

FERNÁNDEZ, O.A. Manejo integrado de malezas. Planta Daninha, v.5, n.2, p.69-75, 1982.

GOMES, S.M.S.; BRUNO, R.L.A. Influência da temperatura e substratos na germinação de sementes de urucum (Bixa orellana L.). Revista Brasileira de Sementes, v.14, n.1, p.47-50, 1992.
GUO, P.; AL-KHATIB, K. Temperature effects on germination and growth of redroot pigweed (Amaranthus retroflexus), Palmer amaranth (A. palmeri), and common waterhemp (A. rudis). Weed Science, v.51, n.6, p.869-875, 2003.

KISSMANN, K.G. Plantas infestantes e nocivas - Tomo I: Plantas inferiores e monocotiledôneas. São Paulo: BASF, 1997. 824p.

LOPES, J.C.; SOARES, A.S. Germinação de sementes de Miconia cinnamomifolia (Dc.) Naud. Brasil Florestal, v.21, n.75, p.31-39, 2003.

MAGUIRE, J.D. Speeds of germination-aid selection and evaluation for seedling emergence and vigor. Crop Science, v.2, p.176-177, 1962.

MAYER, A.C.; POLJAKOFF MAYBER, A. The germination of seeds. 4.ed. Oxford: Pergamon Press, 1989. 270 p.

MURDOCH, A.J.; ROBERTS, E.H.; GOEDERT, C.O. A model for germination responses to alternating temperatures. Annals of Botany, v.63, n.1, p.91-111, 1989.

McDONALD, M.B.; COPELAND, L.O.; KNAPP, A.D.; GRABE, D.F. Seed development, germination and quality. In: MOSER, L. (Ed.) Cool-Season Grass monograph. Madison, Wisc.: American society of Agronomy, 1994.

NASSIF, S.M.L.; PEREZ, S.C.J.G. Efeitos da temperatura na germinação de sementes de amendoim-do-campo (Pterogyne nitens Tul.). Revista Brasileira de Sementes, v. 22, n.1, p.1-6, 2000.

NORONHA, A.; VICENTE, M.; FELIPPE, G.M. Photocontrol of germination of Cucumis anguria L. Biologia Plantarum, v.20, n.4, p281-286, 1978.

POPINIGIS, F. Fisiologia da semente. Brasília, DF: AGIPLAN, 1985, 289p.

SANTOS, S.R.G.; AGUIAR, I.B. Germinação de sementes de branquilho (Sebastiania commersoniana (Baill.) Smith \& Downs) em função do substrato e do regime de temperatura. Revista Brasileira de Sementes, v.22, n.1, p.120-126, 2000 .

SILVA, M.C. Efeito da temperatura na germinação de sementes de manduirana (Senna macranthera (Collad.) Irwin et Barn. - Caesalpiniaceae). Revista Brasileira de Sementes, v.23, n.1, p.92-99, 2001.

SILVA, L.M.M.; RODRIGUES, T.J.D.; AGUIAR, B.A. Efeito da luz e da temperatura na germinação de sementes de aroeira (Myracrodruon urundeuva Allemão). Revista Árvore, v.26, n.6, p.691-697, 2002. 
SOUSA, M.P.S.; BRAGA, L.F.; BRAGA, J.F.; SÁ, M.E.; MORAES, M.L.T. Influência da temperatura na germinação de sementes de sumaúma (Ceiba pentranda (Linn.) Gaertn. Bombacaceae). Revista Brasileira de Sementes, v.22, n.1, p.110-119, 2000.

SOUZA FILHO, A.P.S. Interferência potencialmente alelopática do capim-gengibre (Paspalum maritimum) em áreas de pastagens cultivadas. Planta Daninha, v.24, n.3, p.451-456, 2006.

STECKEL, L.E.; SPRAGUE, C.L.; STOLLER, E.W.; WAX, L.M. Temperature effects on germination of nine Amaranthus species. Weed Science, v.52, n.2, p.217-221, 2004.

TAKAKI, M; HEERING, G.H.; CONE, J.W.; KENDRICK, R.E. Analysis of the effect of light and temperature on the fluence response curves for germination of Rumex obtusifolius. Plant Physiology, v.77, n.3, p.731-734, 1985.
TAYLORSON, R.B.; HENDRICKS, S.B. Phytochrome control of germination of Rumex crispus $\mathrm{L}$. seeds induced by temperature shifts. Plant Physiology, v.50, n.6, p.645-648, 1972.

VARELA, V.P.; FERRAZ, I.D.K.; CARNEIRO, N.B. Efeito da temperatura na germinação de sementes de sumaúma (Ceiba pentandra L.Gaertn. - Bombacaceae). Revista Brasileira de Sementes, v.21, n.2, p.170-174, 1999.

VÁZQUEZ-YANES, C.; OROZCO-SEGOVIA, A. Patterns of seed longevity and germination in the tropical rainforest. Annual Review of Ecology and Systematics, v.24, n.1, p.69-87, 1993. 